\title{
Characteristic signatures of energetic ions upstream from the Kronian magnetosphere as revealed by Cassini/MIMI
}

\author{
Olga E. Malandraki ${ }^{1}$, S. M. Krimigis ${ }^{2,3}$, E. T. Sarris ${ }^{4}$, N. Sergis ${ }^{2}$, \\ K. Dialynas ${ }^{2}$, D. G. Mitchell ${ }^{3}$, D. C. Hamilton ${ }^{5}$ and A. Geranios ${ }^{6}$ \\ ${ }^{1}$ Institute for Astronomy and Astrophysics, National Observatory of Athens, \\ Pedeli, Athens, Greece \\ email: omaland@astro.noa.gr \\ ${ }^{2}$ Office for Space Research and Technology, Academy of Athens, Athens, Greece \\ ${ }^{3}$ Applied Physics Laboratory, Johns Hopkins University, Laurel, Maryland, USA \\ ${ }^{4}$ Democritus University of Thrace, Xanthi, Greece \\ ${ }^{5}$ University of Maryland, Department of Physics and Astronomy, MD, USA \\ ${ }^{6}$ Nuclear and Particle Physics Department, University of Athens, Greece
}

\begin{abstract}
We present unique observations obtained by the Magnetospheric Imaging Instrument (MIMI) on the Cassini spacecraft, of the energetic ion population in the environment upstream from the dawn-to-noon sector of the Kronian magnetosphere during the approach phase and subsequent several orbits of the Cassini spacecraft around the planet. High sensitivity observations of energetic ion directional intensities, energy spectra, and ion composition were obtained by the Ion and Neutral Camera (INCA) of the MIMI instrument complement with a geometry factor of $\sim 2.5 \mathrm{~cm}^{2} \mathrm{sr}$. Charge state information was provided by the Charge-Energy-Mass-Spectrometer (CHEMS) over the range $\sim 3$ to $220 \mathrm{keV}$ per charge. The observations revealed the presence of distinct upstream bursts of energetic hydrogen and oxygen ions up to distances of $\sim 135 R_{S}$. The observations are presented and their theoretical implications are addressed.
\end{abstract}

Keywords. planets and satellites: general, acceleration of particles, plasmas

\section{Introduction}

Energetic charged particle events upstream from planetary bow shocks have been observed at Earth, Jupiter, Saturn and Uranus (e.g., Krimigis 1992). Over the last 3 decades there have been several attempts to interpret the observations in the context of one or more theoretical concepts. Generally the models are divided into those favouring solar wind ions undergoing first-order Fermi acceleration at the bow shock (e.g., Lee 1982; Scholer 1985; Trattner et al. 2003) and those suggesting leakage of pre-accelerated magnetospheric ions into the interplanetary medium (e.g., Krimigis et al. 1985; Anderson 1981; Krupp et al. 2002; Anagnostopoulos et al. 2005).

$\mathrm{O}^{+1}$ has been perceived as an ideal tracer ion of ionospheric origin accelerated to $\mathrm{keV}$ energies in the magnetosphere (Krimigis et al. 1986). Recently, ion composition measurements have provided clear evidence for low-charge-state ions of magnetospheric origin (e.g. $\mathrm{O}^{+1}, \mathrm{~N}^{+1}, \mathrm{O}^{+2}$ ) upstream of the Earth's bow shock (Christon et al. 2000, Posner et al. 2002; Keika et al. 2004). Analysis of the Voyager observations at Jupiter showed that the ions consisted primarily of oxygen and sulphur (Zwickl et al. 1981; Krimigis et al. 1985). Jupiter's magnetosphere with a plasma composition dominated by oxygen and sulphur (Hamilton et al. 1981) emitted from Io's volcanos offered a unique 
opportunity to utilize "tracer ions" to determine the origin of the upstream ions. This composition is clearly different from that of the solar wind.

The existence of energetic ions upstream and downstream of Saturn's bow shock was established during the Voyager 1 and 2 encounters with Saturn in 1980 and 1981, respectively. The energetic ions were found to exist in the interplanetary medium up to distances of $\sim 200 R_{S}$ upstream (Krimigis et al. 1983; Krimigis 1986) from the planet. Analysis of the Voyager 2 observations showed the ion increases extended in energy to $\sim 500 \mathrm{keV}$. Definitive separation of a magnetospheric from a solar wind source has been more difficult than in the case of Jupiter since no unique compositional signature was apparently associated with the magnetosphere of Saturn. In this work, we present unique observations obtained by the Magnetospheric Imaging Instrument (MIMI) on the Cassini spacecraft of the energetic ion population in the environment upstream from the dawn-to-noon sector of the Kronian magnetosphere during the approach phase and subsequent several orbits of the Cassini spacecraft around the planet. These measurements by Cassini/MIMI, which offers unprecedented observational capabilities, are utilized to investigate characteristic signatures of the ion events in terms of the time history of intensities, spectra, composition and charge state of energetic ions upstream from the Kronian Magnetosphere as revealed in the Cassini era. The theoretical implications of the observations are also addressed.

\section{Instrumentation}

High sensitivity observations of energetic ion directional intensities, energy spectra, and ion composition are obtained by the Ion and Neutral Camera (INCA) which is part of the Magnetospheric IMaging Instrument (MIMI) complement on the Cassini spacecraft (Krimigis et al. 2004). MIMI/INCA (which has a $90^{\circ}$ by $120^{\circ}$ field of view) with its large geometry factor $\sim 2.5 \mathrm{~cm}^{2}$ sr in the ion mode is ideal for studies of upstream ion activity, also providing some capability of separating light $(\mathrm{H}, \mathrm{He})$ and heavier $(\mathrm{C}, \mathrm{N}, \mathrm{O})$ ion groups (henceforth referred to as "hydrogen" and "oxygen" respectively). The intensities are integrated over the entire image obtained by INCA. Detailed composition measurements are provided by a second MIMI sensor, the Charge-Energy-Mass-Spectrometer (CHEMS), which is capable of determining independently the charge state, mass, and energy of ions over the range $\sim 3$ to $\sim 220 \mathrm{keV}$ per charge.

\section{Observations and Data Analysis}

The Cassini spacecraft was injected into orbit around Saturn (SOI) on July 1, 2004. Figure 1 (left) presents the orbit of Cassini in an equatorial projection in Saturn Solar Orbit (SSO) coordinates for the period from June 12 (DOY 164) till October 27 (DOY 301 ) in 2004, during its approach phase and subsequent orbits around Saturn. As denoted by the arrow the Sun is to the left of the diagram. Model bow shock and magnetopause curves are presented. For reference the orbits of Titan and Rhea are also shown. The highlighted parts of the Cassini trajectory denote periods during which individual upstream events are analyzed in detail in this work. (For color versions of all figures see online electronic version of the paper).

In Figure 1 (upper right), from top to bottom energetic ion differential intensities versus time are shown in the energy range $3->220 \mathrm{keV}$ as measured by the MIMI/INCA hydrogen channels (H7-H0) for the period DOY 175-180 in 2004, during the approach phase of Cassini, prior to its entry into the magnetosphere of Saturn. This period corresponds to the first period highlighted in Figure 1 (left). On DOY 175, Cassini was at $\sim 90 R_{S}$ radial distance from Saturn. As Cassini approaches Saturn, distinct ions bursts 

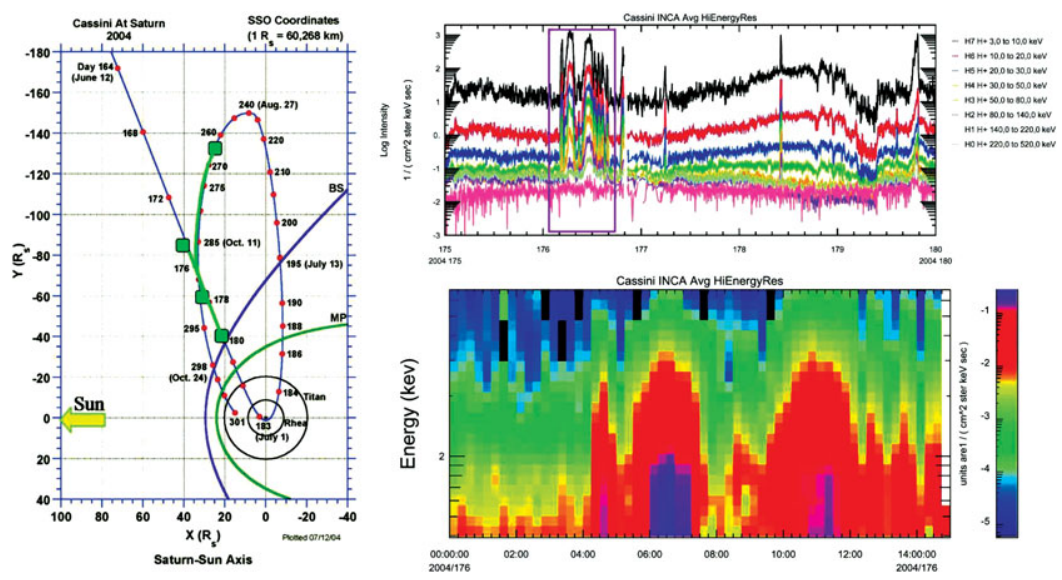

Figure 1. Left: Orbit of Cassini for period DOY 164-301 in 2004. Upper right: Energetic ion intensities $(3->220 \mathrm{keV})$ for DOY 175-180 in 2004. Bottom right: Dynamic spectrogram of intensities measured in the Oxygen channel on DOY 176.
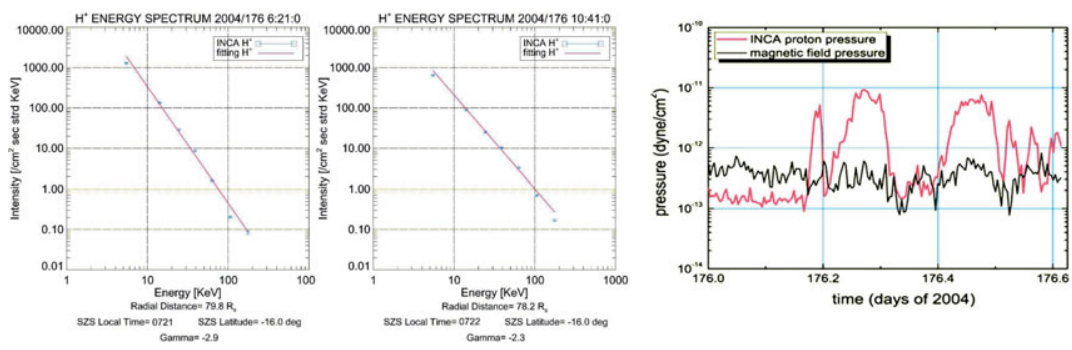

Figure 2. Left: Hydrogen spectra for the events observed on DOY 176. Right: Comparison of the particle and magnetic and field pressures.

are observed by MIMI/INCA. On DOY 179 multiple bow shock crossings started to be observed (Masters et al. 2008). We next focus on a series of upstream ion events delimited by a box in Figure 1 (left), observed by Cassini/MIMI on DOY 176 in 2004 at $\sim 80 R_{S}$.

Figure 1 (bottom right) shows a dynamic spectrogram (Energy vs Time) of intensities measured by the Oxygen channel of INCA from 0000 UT to 1500 UT on DOY 176. The measurements reveal the upstream events near Saturn are also observed in the Oxygen channel up to $\sim 500 \mathrm{keV}$ energy. The Pulse Height Analyzed (PHA) observations available by INCA (not shown) also reveal significant oxygen presence in the upstream events observed on this day. We have also analyzed the magnetic field measurements during this period (not shown) and found evidence for wave structures consistent with ion cyclotron waves for both $\mathrm{H}^{+}$and $\mathrm{O}^{+}$.

In Figure 2 (left panels), energy spectra (assuming protons) at the peak of the events on DOY 176 are shown. The spectra appear to fit well in energy with spectral indices $\gamma$ $\sim 2.9$, and 2.3. An event previously detected near Saturn by Voyager 2 that was examined in detail exhibited a power law of $\sim 2.1$ (Krimigis 1986). Cassini, thus observed somewhat softer spectra during the events on DOY 176. Figure 2 (right panel) shows a comparison of the magnetic field and energetic particle (as derived from the hydrogen channel in the energy range $\sim 3-200 \mathrm{keV}$ ) pressures on DOY 176. During the ion events the particle pressure by far exceeds the magnetic field pressure, with beta ratio values (energetic particle pressure to magnetic field pressure) of $\sim 4-10$. 

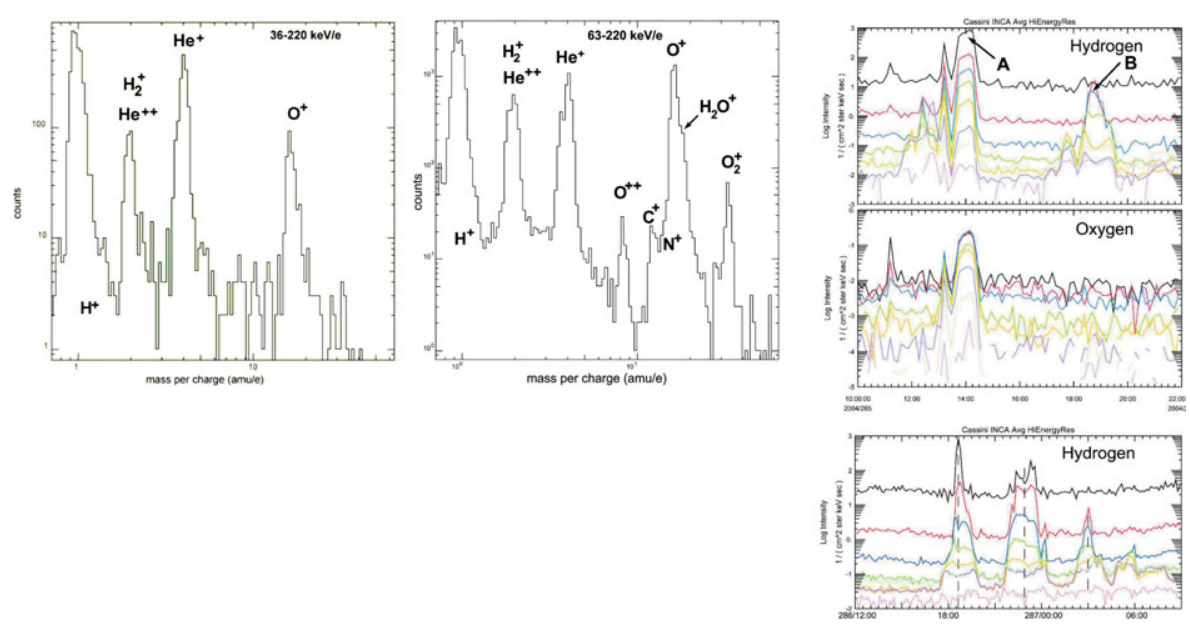

Figure 3. (Left panels) MIMI/CHEMS observations for the periods DOY 242-296 in 2004 (first panel) and DOY 29-41 in 2005 (second panel). (Right, two top panels) INCA/MIMI observations of the upstream events $\sim 134 R_{S}$ on DOY 265 , (Right, bottom panel) INCA/MIMI observations of the upstream events on DOY $286 / 287$.

Figure 3 (left first panel) presents a histogram of counts vs Mass/Charge ratios from the MIMI/CHEMS sensor summed over the period DOY 242-296 in 2004 when Cassini was upstream from the Saturnian bow shock (Masters et al. 2008). The observations reveal that oxygen in the energy range $36-220 \mathrm{keV} /$ charge is present throughout Rev A, from $\sim 150$ to $\sim 50$ Rs upstream. Figure 3 (left second panel) presents composition and charge states of ions as measured by MIMI/CHEMS during Rev C (DOY 29, $\sim 56 R_{S}$, to DOY $41, \sim 43 R_{S}$ in 2005). During this period, Cassini was upstream (Masters et al. 2008) but close to the bow shock. The measurements reveal significant fluxes of $63<\mathrm{E}<220 \mathrm{keV} / \mathrm{e}$ Water group ions $\left(\mathrm{W}^{+}\right)$are present.

The two panels on the right in Figure 3 present differential intensities as measured by the INCA Hydrogen and Oxygen channels from 1000-2200 UT on DOY 265 during upstream events observed by Cassini at $\sim 134 R_{S}$. The PHA observations also showed significant oxygen presence during the first event of this period. The spectrum at the peak of this event (A) appears to fit well a power law in energy with spectral index $\gamma$ $\sim 2.5$. The spectrum of the second event $(B)$ showed a deficiency of low energy ions. A noteworthy feature is that at the end of the first event the particle intensities were observed to decrease abruptly and simultaneously at all energies, in association with an abrupt change in the direction of the magnetic field (not shown), no longer connecting the spacecraft to the planetary bow shock.

The bottom panel on the right in Figure 3 presents differential intensities as measured by the INCA Hydrogen channel from 12:00 UT on DOY 286 to 09:00 UT on DOY 287 in 2004 during upstream events observed by Cassini at $\sim 86$ Rs. PHA observations showed significant oxygen presence during the events. The events are symmetrical around their peak intensities. The start and stop times of high-energy and low-energy ions do not match, with the higher energies evidently observed earlier than the low-energies. The reverse is observed at the end of the event. The observations provide evidence of filaments filled with energetic particles being convected over the spacecraft, with the higher energy particles, which have larger gyroradii, starting to be detected earlier than the lower energy particles which have smaller gyroradii. This is also consistent with the reverse observation as the spacecraft exits the IMF filament. 


\section{Summary and Conclusions}

We have presented preliminary results of the characteristic signatures of energetic ions observed upstream from the Kronian magnetosphere by Cassini/MIMI. Hydrogen ion bursts are observed in the energy range $3-220 \mathrm{keV}$ (and occasionally to $\mathrm{E}>220 \mathrm{keV}$ ) and Oxygen ion bursts in the energy range 32 to $\sim 500 \mathrm{keV}$. The duration of the ion bursts is several minutes up to $4 \mathrm{hrs}$. Some ion bursts are accompanied by distinct diamagnetic field depressions with plasma $\beta \sim 4$ to 10 . There is evidence for wave structures consistent with ion cyclotron waves for both $\mathrm{H}^{+}$and $\mathrm{O}^{+}$. The bursts have a filamentary structure with some exhibiting distinct signatures of 'velocity-filtering effects' at the edges of convecting IMF filaments. MIMI has provided for the first time charge state measurements of upstream ion events at Saturn. The events are of varying composition, with some exhibiting significant fluxes of oxygen. Time-averaged detailed composition measurements upstream show all elements/charge states identified within Saturns MSPH including $\mathrm{O}^{+}, \mathrm{O}^{++}, \mathrm{O}_{2}^{+}$etc. Given that energetic ions trapped within the magnetosphere of Saturn are mostly $\mathrm{H}^{+}, \mathrm{O}^{+}$, and other water group ions $\mathrm{W}^{+}$(Krimigis et al. 2005) we conclude that $\mathrm{O}^{+}$-rich upstream events must be particles leaking from Saturns magnetosphere under favourable IMF conditions. We have thus measured and identified for the first time unique tracer ions at Saturn to discriminate between solar wind and magnetospheric sources. Recent results by the CAPS plasma instrument onboard Cassini (Thomsen et al. 2008) have shown no detectable contribution from magnetospheric $\mathrm{W}^{+}$ ions to upstream suprathermal ions between $3-50 \mathrm{keV} / \mathrm{q}$. This also supports our results on the origin of the particles and strongly suggests the energetic $\mathrm{W}^{+}$group ions observed by Cassini/MIMI leak directly from the magnetosphere and are not produced by energization of lower-energy particles by bow shock-related processes.

\section{References}

Anagnostopoulos, G. C., Efthymiadis, D., Sarris, E. T., \& Krimigis, S. M. 2005, J. Geophys. Res., 110, A10203

Anderson, K. A. 1981, J. Geophys. Res., 86, 4445

Christon, S. P., Desai, M., Eastman, T. E., et al. 2000, Geophys. Res. Lett., 27(16), 2433

Hamilton, D. C., Gloeckler, G., Krimigis, S. M., \& Lanzerotti L. J. 1981, J. Geophys. Res., 86, 8301

Keika, K., Nose, M., Christon, S. P., \& McEntire, R. W. 2004, J. Geophys. Res., 109, A11104

Krimigis, S. M. 1986, in: CNES \& CEPADUES (eds.), Comparative Study of Magnetospheric Systems (France), p. 99

Krimigis, S. M. 1992, Space Sci. Revs, 59, 167

Krimigis, S. M., Zwickl, R. D., \& Baker, D. N. 1985, J. Geophys. Res., 90, 3947

Krimigis, S. M., Carbary, J. F., Keath, E. P. et al. 1983, J. Geophys. Res., 88, 8871

Krimigis, S. M., Sibeck, D. G., \& McEntire, R. W. 1986, Geophys. Res. Lett., 13, 1376

Krimigis, S. M., et al. 2004, Space Sci. Rev., 114, 233

Krimigis, S. M., et al. 2005, Science, 307, 1270

Krupp, N., Woch, J., Lagg, A., et al. 2002, Geophys. Res. Lett., 29, 1736

Lee, M. A. 1982, J. Geophys. Res., 87, 5063

Masters, A., Achilleos, N., Dougherty, M. K., et al. 2008, J. Geophys. Res., 113, A10210

Posner, A., et al. 2002, Geophys. Res. Lett., 29(7), 1099

Scholer, M. A. 1985, in: B. Tsurutani \& R. G. Stone (eds.), Collisionless Shocks in the Heliosphere: A Tutorial Review (Washington, D.C.: AGU), p. 287

Thomsen, M. F., et al. 2008, J. Geophys. Res., 112, A05220

Trattner, K. J., Fuselier, S. A., Peterson, W. K., Chang, S. W., Friedel, R., \& Aellig, M. R. 2003, J. Geophys. Res., 108, 1303

Zwickl, R. D., Krimigis, S. M., Carbary, J. F. et al. 1981, J. Geophys. Res., 86, 8125 


\section{Discussion}

SPANGLER: What processes are occurring which allow ions to escape from the Saturnian magnetosphere into the upstream region?

MALANDRAKI: Processes by which the energetic magnetospheric particles escape from the outer magnetosphere into the Magnetosheath have been discussed for the Earth's case. The particles reach the magnetopause by following magnetospheric drift paths, enter into the magnetosheath and fail to re-enter the magnetosphere (Sibeck et al., JGR 92 (11), 12, 097, 1987; Sarafopoulos et al., JGR, 105 (7), 15, 729, 2000). The loss of magnetospheric particles is caused by finite gyroradius effects. Alternately the energetic magnetospheric particles might escape into the magnetosheath along interconnected magnetosphere magnetosheath magnetic field lines that result from merging (e.g. Speiser and Williams; JGR, 87(4), 2177, 1982)

It has been shown that lower-energy magnetoshealth ions have ready access to the upstream interplanetary medium. The quasi-perpendicular bow shock transition is characterized by 2 downstream ion populations including high-energy gyrating ions in addition to the directly transmitted anisotropic ions. Tanaka et al., JGR, 88(4), 3046, 1983 have shown by particle simulations that this highly anisotropic downstream ion distribution can excite electromagnetic ion cyclotron waves, which, in turn, pitch-angle scatter the gyrating ions in a few ion gyro-periods. As a result, some ions acquire large parallel velocities and move fast enough along the connecting downstream magnetic field to escape back across the bow shock, into the upstream region. Within the model of Edmiston et al., GRL, 9(5), 571, 1982, significant upstream ion fluxes occur for the quasi-parallel portion of the bow shock. The ions are heated and thermalized in a thin layer at the shock front. They calculated the ions returning upstream from a hot Maxwellian distribution at this layer.

Similar processes are apparently taking place in the vicinity of Saturn's magnetosphere.

DAVILA: Is the time variability observed really a time varying process, or are they spatial structures that the spacecraft is passing through?

MALANDRAKI: The upstream ion events exhibit a filamentary structure which strongly suggests we are dealing with spatial structures that the spacecraft is passing through. In some of the events, as presented, signatures of 'velocity filtering effects' at the edges of connecting IMF filaments are observed. 